\title{
Scaling in directed dynamical small-world networks with random responses
}

\author{
Chen-Ping Zhu ${ }^{1,2}$, Shi-Jie Xiong ${ }^{2}$, Ying-Jie Tian ${ }^{3}$, Nan $\mathrm{Li}^{3}$, Ke-Sheng Jiang ${ }^{3}$ \\ ${ }^{1}$ Department of Applied Physics, Nanjing University of Aeronautics and Astronautics, Nanjing, 210016, China \\ ${ }^{2}$ National Laboratory of Solid State Micro-structure and Department of Physics, Nanjing University, Nanjing 210093, China \\ ${ }^{3}$ College of Economics and Management, Nanjing University of Aeronautics and Astronautics, Nanjing 210016, China
}

(October 31, 2018)

\begin{abstract}
A dynamical model of small-world network, with directed links which describe various correlations in social and natural phenomena, is presented. Random response of every site to the imput message are introduced to simulate real systems. The interplay of these ingredients results in collective dynamical evolution of a spin-like variable $S(t)$ of the whole network. In the present model, global average spreading length $\langle L\rangle_{s}$ and average spreading time $\langle T\rangle_{s}$ are found to scale as $p^{-\alpha} \ln N$ with different exponents. Meanwhile, $S$ behaves in a duple scaling form for $N \gg N^{*}$ : $S \sim f\left(p^{-\beta} q^{\gamma} \tilde{t}\right)$, where $p$ and $q$ are rewiring and external parameters, $\alpha, \beta, \gamma$ and $f(\tilde{t})$ are scaling exponents and universal functions, respectively. Possible applications of the model are discussed.
\end{abstract}

PACS numbes: 73.61.Ph,05.60.+w,71.30.+h,72.90.+y

Many natural, social and economic systems, are well described as networks in which sites represent agents and links represent correlations between them (see $[1,2]$ for reviews). Among networks with complex topology, small-world networks (SWN) [3] are based on a highly connected regular lattice in which a fraction $p$ of original links between sites are replaced by randomly rewired links. It has played an important role in the study of real processes [4], such as various problems in the field of physics [5], and those outside it [6-10].

As a platform for understanding processes in systems where SWN topology crucially determines their dynamics, Watts [4], Lago-Fernández et al. [6], Strogatz [9], and, Kuperman and Abramson [10] have analyzed several types of real problems. Activities of individual agents (sites) in their models evolve in time, and couple with each other to induce system response from global coherence, while connections (links) of networks are kept unchanged throughout the process. However, interactions in most social or biological systems are not static and change with time from common knowledge. When states of sites at the ends of a link change with time, correlation between them could correspondingly change. This effect can result in novel dynamical scaling properties which do not appear in previous works.

Suppose an exclusive shop sells a new brand of portable commodity without advertising, this message can be spread out by showing it in the population, and every new buyer can be a source of message, which change his/her role in the crowd in this sale process. Meanwhile, interacting relations among people change continuously, they are not fixed due to everyday activities. Similar things can occur when a contact-infectious disease with all possible incubation (infected-but-not-infective) time propagate through an acquaintance network starting from an index patient. When an infected one finally becomes infective, he changes his role to all others. Sometimes people in incubation periods continuing their social activities even are unaware of infection.

In most information or disease diffusion processes effective interactions are asymmetric or even unidirected, which has been investigated in a lot of literature [11-14]. The direction of a link between a pair of given sites depends on their states. For example, infection passes from infectives to susceptibles, which defines an active link from the former to the later. Meanwhile, any link between two infectives or two susceptibles is inactive in an epidemic spreading. As another example, some special cases of food webs which show small-world effect $[15,16]$, can be described with inherent directed trophic links [11] and their structures can vary with time [12]. These models can illustrate how human pollution or disaster on the environment to be transported up through species at different trophic levels. Recently a non-equilibrium phase transition is displayed in two-dimensional directed SWNs $[17]$.

Usually, individuals in real systems react to input message not simultaneously $[6,10]$. Incubation time of people for the same infectious disease varies depending on their resistivity to it. In parallel, delaying interval from getting a message of the commodity to buying it also vary according to the spending potential of a particular buyer. In one word, there always exist stochastic relaxations or random responses in such dynamical progresses.

In this Letter we attempt to point out that three ingredients mentioned above - dynamically varied interactions, directionality of links, and random responses of agents can interweave together in a real system, and their interplay motivates the present work. We combine these ingredients to form a dynamical directed small-world network (DDSWN) on which we simulate practical spreading processes. With the model dynamical scaling properties of message diffusion and collective response to the message are revealed by numerical simulation and data analysis, which have not been observed from static undi- 
rectional SWN models previously.

The network can be built with the following rules: (1) A rewired one-dimensional (1D) circular lattice with coordination number $2 z$ is constructed. A site labelled $j$ is randomly chosen from all the $N$ sites as the only initial seed in spin-up state, while other sites of indices $i \neq j$ are in spin-down state. So the seed is the first one that is able to send out the message to other sites and to convert spin states of receiving sites. (2) Considering the discrepancies among sites in the response to the message, we define the response time $\tau_{i}$ for every site $i$ as the relaxation period from the receiving of the message to the flipping into the spin-up state. The $\tau_{i}$ 's are random variables obeying distribution probability $P\left(\tau_{i}\right)$. In this paper we consider two types of probability, the uniform and Poisson distributions. The former can be written as

$$
P\left(\tau_{i}\right)=\frac{1}{q} \theta\left(\tau_{i}\right) \theta\left(q-\tau_{i}\right),
$$

where $\theta(x)$ is the step function, being 0 for $x<0$ and 1 for $x \geq 0$, and $q$ is a parameter characterizing the width of the distribution. Here $\tau_{i}$ is uniformly distributed between 0 and $q$, so $q / 2$ is the average response period. For the latter, we use the discrete Poisson distribution

$$
P\left(\tau_{i}\right)=q^{k} e^{-q} / k !
$$

where $k$ is the integer part of $\tau_{i}$, and $q$ corresponds to the average value of $\tau_{i}$. In both distributions parameter $q$ measures the average and diversity of the random response periods. (3) We suppose that the spatial configuration of $\tau_{i}$ is time independent, but connections in SWN are updated in every time unit $\tau_{0}$, i.e., a SWN with new links is produced at every moment $t_{M}=M \tau_{0}$ with $M$ being a positive integer, from the regular 1D circular lattice by rewiring $p$ fraction of bonds. So we have dynamically varied SWNs with the same rewiring probability $p$. (4) All sites evolve their spin states in a parallel way. Once a site of spin-down state is at the end of its relaxation time after receiving the message, it flips onto spin-up state and keeps it, being capable of sending out the same message. Therefore, the links in this dynamical SWN are directional as the message can only be transmitted from a spin-up site to a spin-down site, and the transmission from a spin-down site or to a spin-up site is ineffective. Actually, the direction of a link reflects the irreversible nature in diffusion processes, such as the spreading of pollution in food webs $[9,11]$ and diffusion in directed percolation models [18].

For an undirected and static SWN, the smallworld effect can be described by the characteristic path length $\bar{l}$, i.e., the averaged shortest distance between any two sites. It follows the scaling law $[1,2]$,

$$
\bar{l}(N, p) \sim\left(N^{*}\right)^{1 / d} F\left(N / N^{*}\right),
$$

where the scaling function $F(u)$ has the limits $F(u) \sim$ $u^{1 / d}$ for $u \ll 1, F(u) \sim \ln u$ for $u \gg 1$, and $N^{*} \sim p^{-1}$ is a crossover size separating the big- and small-world regimes. In DDSWN, however, not all sites are effectively connected with each other since only links from sites in spin-up state to those in spin-down state are active. Therefore, $\bar{l}$ loses its original meaning in the present model. In order to investigate how wide and how fast the message spreads out in DDSWNs, we introduce the time-dependent average spreading length, $\left\langle L_{\mu}\right\rangle \equiv \sum_{\nu=1}^{\mu} n_{\nu} l_{\nu} / \sum_{\nu=1}^{\mu} n_{\nu}$, and the average spreading time, $\left\langle T_{\mu}\right\rangle \equiv \sum_{\nu=1}^{\mu} n_{\nu} t_{\nu} / \sum_{\nu=1}^{\mu} n_{\nu}$, where $\mu$ is the time index, $t_{\mu}=\mu t_{0}, n_{\mu}$ is the number of receivers created between the $(\mu-1)$ th and the $\mu$ th moments, and $l_{\mu}$ is the average length from the initial seed to these receivers. We calculate them by numerical simulations in which the data are obtained by ensemble average over at least 50 random configurations with different $\left\{\tau_{i}\right\}$ and location of the initial seed. The obtained results are shown in Figs. 1 and 2 .

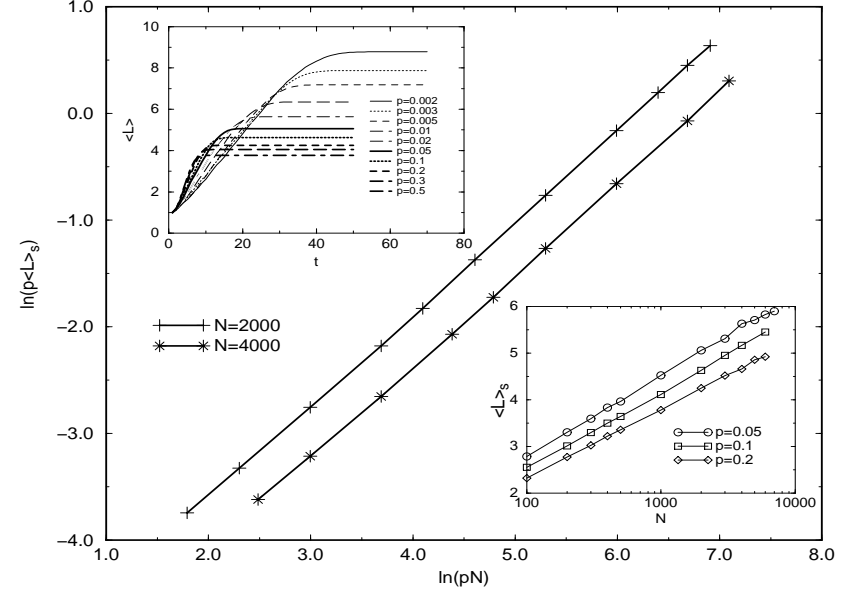

FIG. 1. Log-log plot of saturated average path length $\langle L\rangle_{s}$, in the case of uniform distribution of $\tau_{i}$, versus $p$. Two lines have the same average slope, 0.86. Upper-left inset: $\langle L\rangle$ as a function of $t$ for the curve with symbols $(+)$ in the main panel. Lower-right inset: log-linear relation of $\langle L\rangle_{s}$ versus $N$. Other parameters for these curves are $z=5$ and $q=10$, and thedime is in units to.

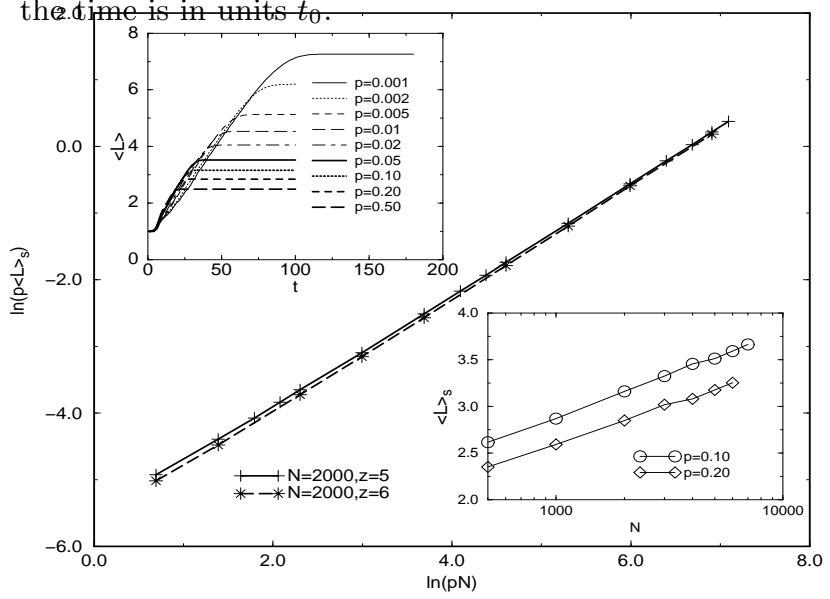

FIG. 2. The same as those in Fig. 1, but for Poisson distribution of $\tau_{i}$. In this case the average slope of lines in the main panel is 0.84. $q=10$ and other parameters are indicated in the figure. 
It is found that for given $N$ and $z$ the average path length $\langle L(t)\rangle$ (here we indicate the time-dependence explicitly and omit the suffix $\mu$ ) increases monotonically in time towards its $p$-dependent saturation value $\langle L\rangle_{s}$ (see the upper-left insets of Figs. 1 and 2), which shows the finite-size effect of $p$. Summation over $n_{\nu}$ in the calculation of $\langle L\rangle_{s}$ tells us that eventually all sites will be successively visited by the message sooner or later, suggesting the higher adaptability of the dynamical models in description of the diffusion processes compared with the static directed SWN models [14]. We will use $\langle L\rangle_{s}$ and $\langle T\rangle_{s}$ to characterize global properties of the networks, since in saturation the quantities are no longer time dependent. Compared with the scaling relation Eq. (3) for $\bar{l}(N, p)$ in the static SWNs, the corresponding quantity $\langle L\rangle_{s}$ in the DDSWNs scales with $\ln N$ for given $p$ (see the lower-right insets of Figs. 1 and 2), but it depends on $p$ in a power law $\langle L\rangle_{s} \sim p^{-\alpha_{L}}$ for a fixed $N$ (see the main panels of Figs. 1 and 2, where the slope of straight lines gives the value $1-\alpha_{L}$ ). Therefore, we have a new scaling formula

$$
\langle L\rangle_{s} \sim p^{-\alpha_{L}} \ln N,
$$

where $\alpha_{L}=0.14 \pm 0.02$ for uniform distribution and $\alpha_{L}=0.160 \pm 0.005$ for Poisson distribution, extracted from the data in Figs. 1 and 2, respectively. Meanwhile, the average spreading time $\langle T\rangle_{s}$ behaves in the same form as $\langle L\rangle_{s}$, but with different parameters. We obtain $\alpha_{T}=0.29 \pm 0.01$ and $\alpha_{T}=0.245 \pm 0.006$ for uniform and Poisson distributions, as extracted from the data in Figs. 3 and 4, respectively. The message from the initial seed passes through tree-like paths, and $\langle L\rangle_{s}$ and $\langle T\rangle_{s}$

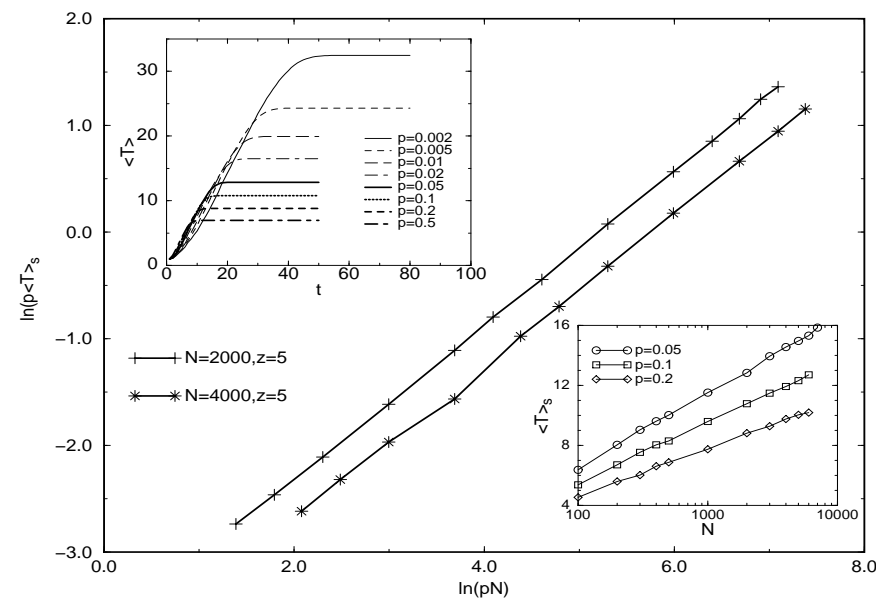

FIG. 3. Saturated average spreading time $\langle T\rangle_{s}$ as a function of $p$ for the uniform distribution of the response time. Other parameters are the same as those in Fig. 1. The average slope of the lines in the main panel is 0.81 .

are the maximal path length and spreading time by which the message reaches the final sites. The scaling relations of $\langle L\rangle_{s}$ and $\langle T\rangle_{s}$ are unique features of DDSWN model. The simulations suggest that the $p$-dependence of the saturated $\langle L\rangle$ and $\langle T\rangle$ is attributed to the dynamically varied connections and the finite-size effect, the factor $\ln N$ in the scaling relation can be attributed to the geometrical characteristics of the SWN background, and the factor $p^{-\alpha_{L}}$ is originated from the diffusion mechanism of the directional links, distinctive in comparison with the behavior of $\bar{l}$ in the ordinary SWNs. The exponents $\alpha_{L}$ and $\alpha_{T}$ still depend on specific form of the distributions of the relaxation time. On the other hand, as it should be anticipated, $\langle L\rangle_{s} \sim N$ for $N \ll N^{*}$ in the big-world regime.

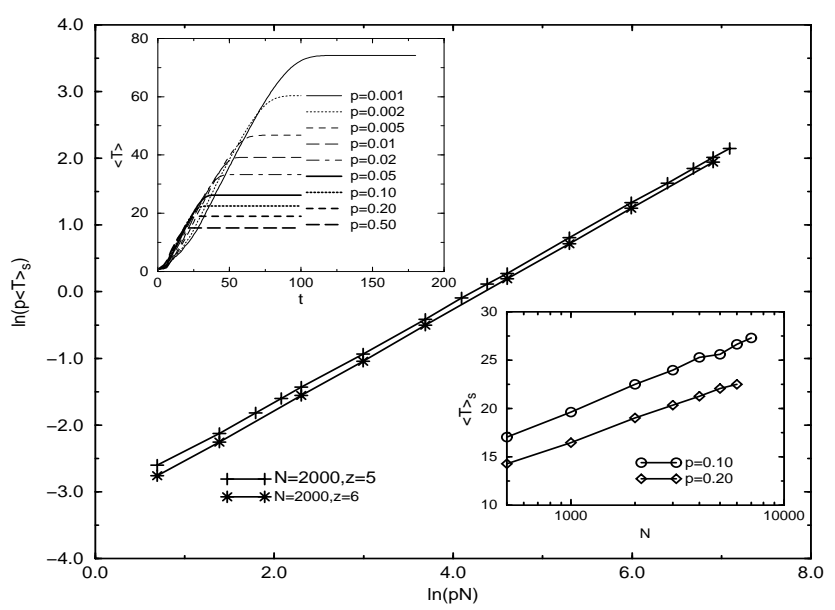

FIG. 4. The same as those in Fig. 3, but for the Poisson distribution of the response time. Other parameters are the same as those in Fig. 2. The average slope of the lines in main panel is 0.755 .

We now turn to the behavior of $S(t)$, defined as the number of all sites in spin-up states at moment $t$ divided by $N$. It represents the ratio of the sites in spin-up state, and its temporal derivation, $S^{\prime}(t)$, reflects the probability of a spin-down site to be flipped into the spin-up state in a unit of time at moment $t$. Because in this model all the sites should be in spin-up state after an enough time, $S(t) \rightarrow 1$ at the limit $t \rightarrow \infty$. We numerically calculate $S(t)$ for various values of parameters. The results for different values of $p$ and fixed $q$ in the case of uniform distribution are plotted in Fig. 5(a). All the curves can merge together if the time is rescaled as $t_{\mathrm{sc}}=\left(p / p_{0}\right)^{\beta} t$, as can be seen from Fig. 5(b) in which we use $p_{0}=0.1$ and $\beta=0.20 \pm 0.005$ is extracted. The time rescaling is done for the Poisson distribution, as shown in Figs. 5(c) and $5(\mathrm{~d})$, and we get $\beta=0.186 \pm 0.006$. At the same time, if $q$ is varied as well, we can also use the time rescaling, $t_{\mathrm{sc}}^{\prime}=\left(q / q_{0}\right)^{-\gamma} t_{\mathrm{sc}}$ to merge the curves, as shown in Fig. 6 . From this we extract the exponent $\gamma=0.750 \pm 0.003$ for the uniform distribution and $\gamma=0.880 \pm 0.006$ for the Poisson distribution, both with $q_{0}=10$. Thus, we have the following scaling form of $S(t)$ : 


$$
S(t) \sim S\left(p^{-\beta} q^{\gamma} \tilde{t}\right) \equiv f(\tilde{t}),
$$

where $\tilde{t}=p_{0}{ }^{-\beta} q_{0}{ }^{\gamma} t_{\mathrm{sc}}^{\prime}$ and $f(\tilde{t})$ is a universal function for a given distribution.
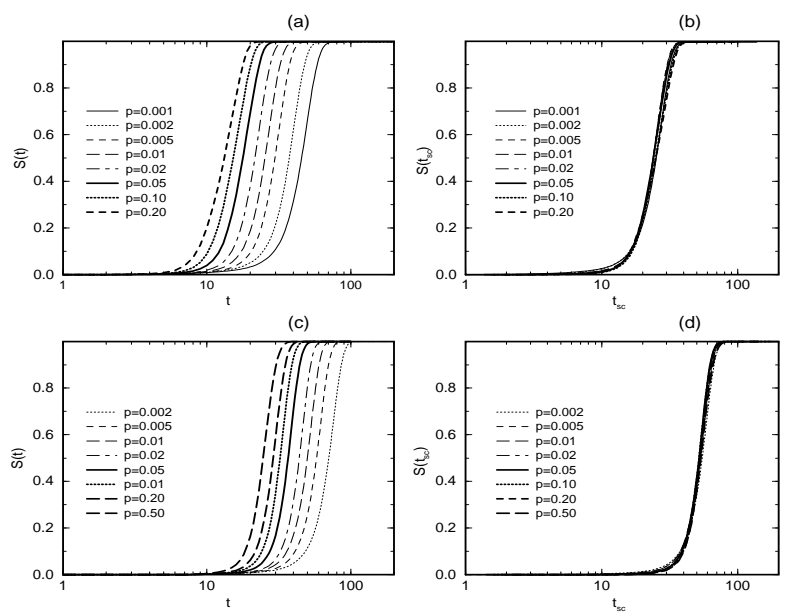

FIG. 5. The ratio of spin-up sites versus time $t$ (a and $\mathrm{c}$ ) and versus rescaled time $t_{\mathrm{sc}}=\left(p / p_{0}\right)^{\beta} t$ (b and $\mathrm{d}$ ) for the uniform ( $\mathrm{a}$ and $\mathrm{b}$ ) and Poisson (c and d) distributions. $p_{0}=0.1$ is used. Other parameters are: $N=2000, z=5$, and $q=10$.
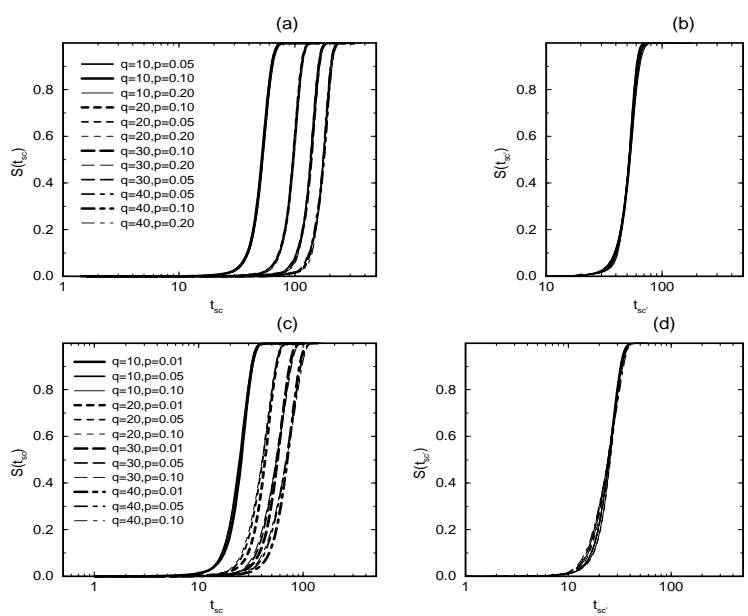

FIG. 6. The ratio of spin-up sites versus $t_{\mathrm{sc}}$ (a and c) and versus $t_{\mathrm{sc}}^{\prime}=\left(q / q_{0}\right)^{-\gamma} t_{\mathrm{sc}}(\mathrm{b}$ and $\mathrm{d})$ for Poisson ( $\mathrm{a}$ and $\mathrm{b}$ ) and the uniform (c and d) distributions. $q_{0}=10$ is used. Other parameters are: $N=2000$ and $z=5$.

The distributions of the response time used here are phenomenological functions reflecting the nature of the DDSWN. Other forms can also be used to describe specific systems. The results of our simulations with different distributions suggest that there exists common scaling behavior of the diffusion dynamics in DDSWNs and only the values of exponents depend on the form of distribution. For a specific process, the response time has a definite meaning. Sales problem of a new type of commodity, for example, $\tau_{i}$ reflects the delaying time between the awareness and the buying of the commodity for a consumer, and $q$ is related to the discrepancy in consumptive potential among them. Food webs, as another example, usually have a few directed links [16] from the basal species to the top ones, and moreover, have average trophic distance scaling in $\ln N$ [19]. All of them share typical values of $\langle L\rangle_{s}$ in the DDSWN model. This implies that the model can capture the essential feature of dynamical directionality of food webs although the smallworld effect in this problem is still in dispute $[15,16]$. By the way, on revision of our submitted manuscript, similar $\ln N$ scaling behaviors of SWNs were independently found in quantum and classical diffusion problems [20].

This work is supported by China Aeronautic Science Fundation under Grant No.00J52079.

[1] Reka Albert, Albert-Laszlo Barabási, Rev. Mod. Phys. 74, 47 (2002), and references therein.

[2] S. N. Dorogrovtsev, J. F. F. Mendels, Adv. Phys. 51, 1079 (2002), and references therein.

[3] D. J. Watts, S. H. Strogatz, Nature, 393, 440 (1998).

[4] D. J. Watts, Small Worlds,(Princeton University Press, Princeton. NJ, 1999).

[5] A. Barrat and M. Weigt, Eur. Phys. J. B 13, 547 (2000). A. Scala, L. A. N. Amaral and M. Barthélémy, Eur. Phys. Lett. 55, 594 (2001). Chen-Ping Zhu and ShiJie Xiong, Phys. Rev. B 62, 14780 (2000); and ibid63, 193405 (2001). S. Jespersen, I. M. Sokolov and A. Blumen, Phys. Rev. E 62, 4405 (2000). C. P. Herrero, Phys. Rev. E 65, 066110 (2002).

[6] L. F. Lago-Fernández, et al. Phys. Rev. Lett.84, 2758 (2000).

[7] C. Moore and M. E. J. Newman, Phys. Rev. E 61, 5678 (2000).

[8] P. Svenson and D. A. Johnston, Phys. Rev. E 65, 036105 (2001).

[9] S. H. Strogatz, Nature 410, 268 (2001).

[10] M. Kuperman and G. Abramson, Phys. Rev. Lett.86, 2909 (2001).

[11] M. E. J. Newman, Phys. Rev. E 66, 016128 (2002); S. L. Pimm, et al. Nature, 350, 669 (1991).

[12] K. O. Winemiller, Am. Nat. 134, 960 (1989); R. L. Kitching, Oikos, 48, 280 (1987).

[13] H. Jeong, et al. Nature (London) 407, 651 (2000); P. B. Jayanth, et al. Nature, 399, 130 (1999).

[14] A. Ramzanpour and V. Karimipour, Phys. Rev. E 66, 036128 (2002)

[15] J. M. Montoya and R. V. Solé, J. Theor. Biol. 214, 405(2002).

[16] R. J. Williams, et al. Proc. Natl. Acad. Sci. USA 99,12913 (2002). J. A. Dunne, et al. ibid 12917 (2002); D. Garlachelli, et al. Natue, 423, 165 (2003).

[17] A. D. Sanchez, et al. Phys. Rev. Lett.88, 048701 (2002).

[18] Y. Y. Goldschmidt, et al. Phys. Rev. E 59,6381 (1999).

[19] J. Camacho, et al. Phys. Rev. Lett. 88,228102 (2002).

[20] B. J. Kim, et al. Phys. Rev. B 68, 014304 (2003). 